\title{
Necrotising granulomas of the uterine corpus
}

\author{
A B Akosa, F Boret
}

\begin{abstract}
Necrotising granulomatous inflammation of the uterine corpus associated with transcervical laser ablation of the endometrium occurred in four patients. The abnormalities seen, including extensive necrosis and hyalinisation with foreign body giant cells containing black foreign material, and eosinophilic homogenisation around blood vessels, were due to the effects of tissue fulguration.

The presence of black foreign material in loosely organised histiocytes should alert the pathologist even in the absence of clinical information; stains for organisms are negative.
\end{abstract}

(F Clin Pathol 1993;46:953-955)

Necrotising granulomas of non-infectious origin are rare and occur mostly in association with avasculitis. Cases have been reported in several sites including prostate, ${ }^{1}$ fallopian tube, $^{2}$ cervix $^{3}$ kidney $^{4}$ and peritoneum ${ }^{5}$ following tissue fulguration during minimal invasive surgery. Four cases have been reported so far in the endometrium, two of which were after hysterotomy and laparoscopic diathermy resection ${ }^{6}$; two were after laser ablation..$^{78}$

We report four cases involving both endometrium and myometrium after trans-cervical resection of the endometrium using a neodymium:yttrium-aluminium-garnet $\mathrm{Nd}$ YAG) laser

\section{Case reports}

CASE 1

A 33 year old woman presented with a two year history of menorrhagia producing clots but no flooding, and dysmenorrhoea. Her periods were regular. Dilatation and curettage was performed and she was given Primolut $\mathrm{N}$ but the symptoms persisted. She was then given Danazol before ablation. The endometrial ablation was performed with an Nd-YAG laser following transcervical hysterotomy. The period of amenorrhoea lasted for six days with recurrence of vaginal bleeding, gradually increasing to blood clots. A total abdominal hysterectomy was performed three months after the ablation. The uterus and cervix measured $8.5 \times 5 \times 3 \mathrm{~cm}$ and the endometrium was replaced by exuberant necrotic and haemorrhagic tissue. The myometrium and cervix appeared normal macroscopically.
CASE 2

A 35 year old woman presented with very heavy vaginal bleeding two weeks after a termination of pregnancy and sterilisation. She was given Danazol before endometrial ablation, which was performed eight months later through a transcervical hysterotomy using an Nd-YAG laser. She had a period of amenorrhoea lasting for almost two years. The bleeding restarted and 30 months after the ablation she had a hysterectomy. The uterus and cervix measured $8 \times 5 \times 3 \mathrm{~cm}$ and the cut surface showed an obliterated and fibrosed endometrial cavity. The myometrium and cervix appeared normal.

CASE 3

A 48 year old woman with a one year history of irregular, prolonged, heavy periods with flooding and clots and dysmenorrhoea had dilatation and curettage which showed incipient menstrual endometrium. The symptoms persisted, leading to a transcervical endometrial resection with the Nd-YAG laser. Postablation amenorrhoea lasted only one week and heavy vaginal bleeding and lower abdominal pain recurred requiring a hysterectomy after five months. The uterus and cervix measured $9 \times 5.5 \times 4 \mathrm{~cm}$ and showed no clinically important abnormality macroscopically.

\section{CASE 4}

A 32 year old woman with an eight year history of menorrhagia had an unsuccessful dilatation and curettage. She was given Danazol for six weeks before endometrial ablation, which was performed using the NdYAG laser. After ablation amenorrhoea developed but lasted for only three months with recurrence of heavy bleeding and abdominal pain. A total abdominal hysterectomy was performed 15 months after the ablation. The uterus and cervix measured $7.5 \times 4.5 \times 3 \mathrm{~cm}$ and appeared normal macroscopically.

\section{Pathology}

The uterine specimens from all four patients were processed conventionally and stained with haematoxylin and eosin for routine histological examination. A representative block from each case was cut and stained with periodic acid Schiff reagent, Grocott's silver stain for fungi, Ziehl Neelsen for acid fast bacilli, Perls's reagent for iron, elastic van Gieson, Gordon and Sweet's technique for reticulin and Lendrum's Martius scarlet blue (MSB) for fibrin. 


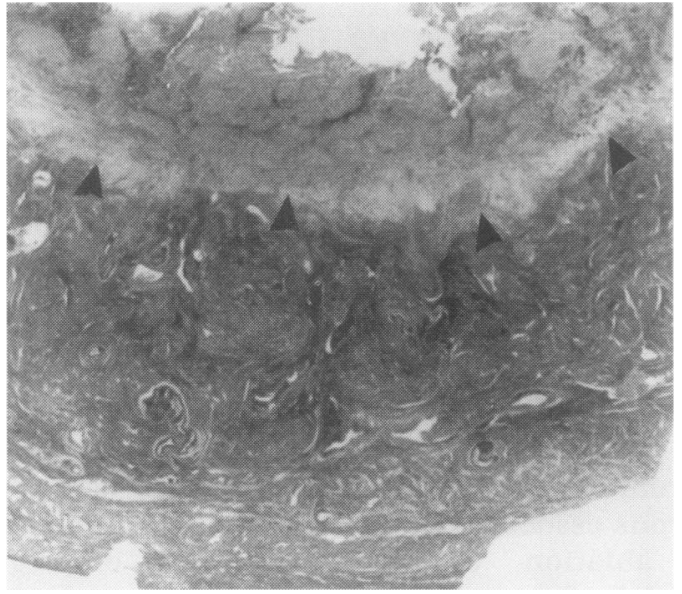

Figure 1 Low power photomicrograph of the endomyometrial section show total necrosis of the endometrium and hyalinisation of the endomyometrial junction. The base of the lesion is well delineated (arrowheads).

The abnormalities found affected the endometrium either completely (fig 1) or focally. The myometrium was involved superficially in all cases with delineation of the base of the lesion. The endometrium contained eosinophilic necrotic debris with larger foci of hyalinisation and ghost outlines of blood vessels. These patches of hyalinisation extended focally up to $0.2 \mathrm{~cm}$ away from the base of the lesion. The necrotic areas were surrounded by an inflammatory infiltrate of lymphocytes, plasma cells, histiocytes, haemosiderin-laden macrophages and multinucleate giant cells of the Langhans and foreign body type, some of which contained black and yellow-brown material (fig 2). Superficial myometrial vessels were lined completely by an eosinophilic, structureless material reminiscent of fibrinoid degeneration.

Reticulin staining outlined fibres within the hyalinised patches and the elastic stain indicated coagulation of elastic tissue around the blood vessels, producing the eosinophilic homogenisation seen on the haematoxylin and eosin stain. MSB for fibrin around the blood vessels was negative. The elastic stain also outlined the site of vessels within the necrotic areas. Iron deposits were present in macrophages and giant cells. All stains for infective organisms were negative.

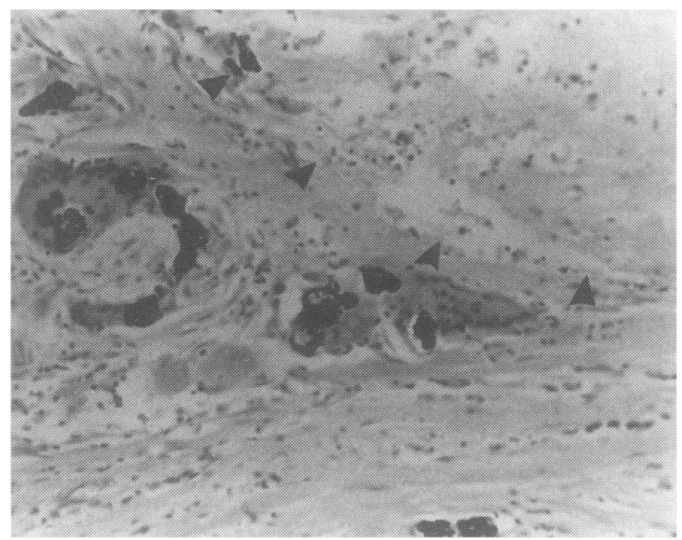

Figure 2 Foreign body giant cells containing black foreign material in the necrotic endometrium. Necrosis is seen on the upper right hand corner (arrowheads).

\section{Discussion}

Transcervical resection of the endometrium is a hysteroscopic method of endometrial ablation in which the endometrium is coagulated, vaporised, or excised under direct vision, with a laser and electrocautery or radiofrequency energy. This form of minimal invasive surgery offers an alternative to hysterectomy in women with menorrhagia refractory to the other forms of treatment.

In a multicentre study of 859 women treated for menorrhagia by Nd-YAG laser ablation of the endometrium, no major complications occurred. ${ }^{9}$ Goldrath, in a 10 year follow up study, reported a $93 \%$ success and a small number of complications including fluid overload, bleeding, urinary tract infection, and a single case each of cervical bleeding, endomyometritis, and uterine perforation. ${ }^{10}$ In the latter case the laser ablation was stopped at the time, and two months later the patient returned to hospital for the procedure to be completed. The case of endomyometritis was asymptomatic except for purulent discharge which was successfully treated with antibiotics. This differs from our cases because all of them had a recurrence of their symptoms and required a hysterectomy. Subsequently, however, deaths have been described following Nd-YAG laser ablation, all of which were attributed to the coaxial fibre tip cooled either by $\mathrm{CO}_{2}$ or $\mathrm{N}_{2}$ gas. ${ }^{112}$ The cause of death in these women was due to air embolism. The use of a bare fibre with no coaxial channel and cooling by external fluid has prevented further fatalities.

The granulomas found differed from infectious or sarcoid type granulomas as they were poorly formed and contained black foreign material but no organisms. They also differed from the rheumatoid-like granulomas described following previous surgery in the prostate ${ }^{1}$ by the lack of a linear configuration. The necrotising granulomas and noticeable patchy hyalinisation of the myometrium, and in particular, hyalinisation around blood vessels are due to tissue fulguration. There was no vascular occlusion and the lesions could not therefore be attributed to local hypoperfusion. Of particular interest was the coagulation of elastic tissue around the blood vessels and the hyalinised areas in the myometrium. These abnormalities extended focally beyond the base of the lesion. Similar non-degradable black foreign material seen in the peritoneum following Nd-YAG laser use has been analysed by electron diffraction and shown to contain carbon and aluminium oxide materials from which the laser probe was made. ${ }^{7}$

Many transcervical resections of the endometrium have been performed but to date only eight cases (including our study) have been reported showing necrotising granulomas of the uterine corpus. This would confirm the assertion that the necrotising granulomatous response to tissue fulguration represents an idiosyncratic response.

We are grateful to Drs McMillan and Baldwin for allowing us to report on their patients, to Dr Thomas for her examination of one of the cases, and to Dr Owen for his comments on the manuscript. 
1 Lee G, Shepherd N. Necrotising granulomata in prostatic resection specimens-a sequel to previous operation. f Clin Pathol 1983;36:1067-70.

2 Roberts JT, Roberts GT, Maudsley FR. Indolent granulomatous necrosis in patients with previous tubal diathermy. Am ₹ Obstet Gynecol 1977;129:112-3.

3 Evans CS, Klein HZ, Goldman RL, Kohout ND. Necrobiotic granulomas of the uterine cervix: a probNecrobiotic granulomas of the uterine cervix: a probable posto

4 Balogh $\mathrm{K}$. Pallisading granuloma in the kidney after open biopsy. Am $₹$ Surg Pathol 1986;10:441-2.

5 Clarke TJ, Simpson RHW. Necrotizing granulomas of peritoneum following diathermy ablation of endometriosis. Histopathology 1990;16:400-2.

6 Ashworth MT, Moss CI, Kenyon WE. Granulomatous endometritis following hysteroscopic resection of the endometrium. Histopathology 1991;18:185-7.

7 Thurrell W, Reid P, Kennedy A, Smith JHF. Necrotizing granulomas of the peritoneum. Histopathology 1991;18:190. Ferryman SR. Necrotizing granulomatous endometritis following endometrial ablation therapy. $\mathrm{Br} \mathcal{F}$ Obstet Gynaecol 1992;99:928-30.

9 Garry R, Erian J, Grochmal SA. A multicentre collaborative study into the treatment of menorrhagia by NdYAG laser ablation of the endometrium. Br $f$ Obstet Gynaecol 1991;98:357-62.

10 Goldrath $\mathrm{MH}$. Intrauterine laser surgery. In: Keye WR ed. Laser surgery in gynecology and obstetrics. Chicago: Year Book Medical Publishers, Inc 1989:151-65.

11 Baggish MS, Daniel JF. Death caused by air embolism associated with neodymium-yttrium-aluminum-garne laser surgery and artificial sapphire tips. Am $\mathcal{F}$ Obstet Gynecol 1989;161:877-8.

12 Baggish MS, Daniel JF. Catatrophic injury secondary to the use of coaxial gas-cooled fibres and artificial sapphire tips for intra-uterine surgery. Lasers Surg Med 1989;9:581-4.

\title{
$\mathrm{T}$ cell lymphoid aggregates in bone marrow in idiopathic hypereosinophilic syndrome
}

\author{
J Metz, K M McGrath, H F Savoia, C G Begley, R Chetty
}

\begin{abstract}
Idiopathic hypereosinophilic syndrome (HES) comprises a heterogeneous group of disorders characterised by prolonged eosinophilia with no obvious cause. A patient with longstanding HES is reported in whom unusual non-neoplastic peritrabecular lymphoid aggregates were present in the bone marrow, a hitherto undescribed association, as far as is known. An eosinophil colony stimulating activity was detected in the serum. The findings in this patient provide further evidence for an important role for eosinophil colony stimulating activity interleukin-5 mediated $T$ lymphocyte control of eosinophil production in the pathogenesis of the HES.
\end{abstract}

$(\mathfrak{F}$ Clin Pathol 1993;46:955-958)

Idiopathic hypereosinophilic syndrome (HES) includes a heterogeneous group of disorders in which there is prolonged eosinophilia of undetectable cause and various organs affected. Interleukin 5 (IL-5), a product of activated $T$ lymphocytes, is an important cytokine involved in the production of eosinophils. ${ }^{1}$ Increased IL-5 concentrations have been reported in patients with HES, prompting the suggestion that excessive quantities of IL-5 may account for the characteristic eosinophilia. ${ }^{23}$ We report a patient with longstanding HES in whom unusual, non-neoplastic peritrabecular $\mathrm{T}$ cell lymphoid aggregates were present in the bone marrow, an association, which, to our knowledge, has not been recorded before.

\section{Case report}

The patient was a 58 year old male spray painter. He originally came from Sicily and had been in Australia since 1958. He had been well until 1978, when, aged 44 , he was admitted to hospital with a deep venous thrombosis. Hepatomegaly and spleen palpable $2 \mathrm{~cm}$ from the costal margin were noted. Blood examination showed a total leucocyte count of $10.1 \times 10^{9} / 1$, with a pronounced eosinophilia of $2.7 \times 10^{9} / 1$. A bone marrow aspirate indicated hypercellular particles, with increased numbers of eosinophils and eosinophil precursors. A marrow trephine biopsy was not carried out at that time. A liver biopsy specimen showed portal fibrosis with a dense infiltration of eosinophils in the portal tracts. No cause for the pronounced eosinophilia could be established, and HES was diagnosed.

The patient presented to the Royal Melbourne Hospital in May 1992 for the investigation of a heart murmur. Examination identified pityriasis versicolor of the skin, an enlarged liver, and a spleen palpable $3 \mathrm{~cm}$ below the costal margin. There was no lymphadenopathy. Cardiac investigation revealed mitral and tricuspid incompetence; a myocardial biopsy specimen showed histological features consistent with endocardial fibrosis. A skeletal survey indicated no abnormality.

Blood examination showed a haemoglobin value of $139 \mathrm{~g} / \mathrm{l}$, a platelet count of $124 \times$ $10^{9} / 1$, and leucocytes at $12 \cdot 1 \times 10^{9} / 1$, with neutrophils $4.59 \times 10^{9} / 1$, monocytes $0.84 \times$ $10^{9} / 1$, and lymphocytes $1.93 \times 10^{9} / 1$. Pronounced eosinophilia was present, the eosinophils numbering 4.71 $\times 10^{9} / 1$. Many of the eosinophils showed areas of cytoplasmic hypogranulation. Serum vitamin $B_{12}$ was greater than $1800 \mathrm{pg} / \mathrm{ml}$ and the serum $\operatorname{IgE}$ titre was normal. A bone marrow aspirate and a trephine biopsy specimen were taken.

Investigations aimed at establishing a cause for persistent eosinophilia were all negative. Antibodies to Schistosoma mansoni and Toxocara canis were not detected by enzyme immunoassay. No parasites were detected in 